\title{
Topological quantum fluctuations in lattice QCD with overlap Dirac quarks
}

\author{
JLQCD and TWQCD Collaborations: T.H. Hsieh*1, S. Aoki ${ }^{2,3}$, T.W. Chiu ${ }^{4,5}$, \\ H. Fukaya ${ }^{6}$, S. Hashimoto ${ }^{7,8}$, T. Kaneko ${ }^{7,8}$, H. Matsufuru ${ }^{7}$, J. Noaki ${ }^{7}$, K. Ogawa ${ }^{4}$, \\ T. Onogi ${ }^{9}$, N. Yamada ${ }^{7,8}$ \\ ${ }^{1}$ Research Center for Applied Sciences, Academia Sinica, Taipei 115, Taiwan \\ ${ }^{2}$ Graduate School of Pure and Applied Sciences, University of Tsukuba, Tsukuba 305-8571, \\ Japan \\ ${ }^{3}$ Riken BNL Research Center, Brookhaven National Laboratory, Upton, NY11973, USA \\ ${ }^{4}$ Department of Physics, and Center for Theoretical Sciences, National Taiwan University, \\ Taipei 10617, Taiwan \\ ${ }^{5}$ Center for Quantum Science and Engineering, National Taiwan University, Taipei 10617, \\ Taiwan \\ ${ }^{6}$ Department of Physics, Nagoya University, Nagoya 464-8602, Japan \\ ${ }^{7}$ High Energy Accelerator Research Organization (KEK), Tsukuba 305-0801, Japan \\ ${ }^{8}$ School of High Energy Accelerator Science, The Graduate University for Advanced Studies \\ (Sokendai), Tsukuba 305-0801, Japan \\ ${ }^{9}$ Department of Physics, Osaka University Toyonaka, Osaka 560-0043, Japan
}

\begin{abstract}
We determine the topological susceptibility $\left(\chi_{t}\right)$ and the second normalized cumulant $\left(c_{4}\right)$ in the topologically-trivial sector $\left(Q_{t}=0\right)$ generated by lattice QCD simulations with overlap Dirac quarks, for $N_{f}=2$ and $N_{f}=2+1$, respectively. The $\chi_{t}$ and $c_{4}$ are extracted from the plateau (at large time separation) of the 2-point and 4-point time-correlation functions of the flavor-singlet pseudoscalar meson $\left(\eta^{\prime}\right)$, which arises from the finite size effect due to fixed topology. In the small $m_{q}$ regime, our result of $\chi_{t}$ and $c_{4}$ both agree with the chiral effective theory.
\end{abstract}

The XXVII International Symposium on Lattice Field Theory - LAT2009

July 26-31 2009

Peking University, Beijing, China

\footnotetext{
${ }^{*}$ Speaker.
} 


\section{Introduction}

In quantum chromodynamics (QCD), the topological susceptibility $\left(\chi_{t}\right)$ is the most crucial quantity to measure the topological charge fluctuations of the QCD vacuum, which plays an important role in breaking the $U_{A}(1)$ symmetry. Theoretically, $\chi_{t}$ is defined as

$$
\chi_{t}=\int d^{4} x\langle\rho(x) \rho(0)\rangle=\frac{\left\langle Q_{t}^{2}\right\rangle}{\Omega}, \quad Q_{t} \equiv \int d^{4} x \rho(x),
$$

where $\Omega$ is the volume of the system, $Q_{t}$ is the topological charge (which is an integer for QCD), and $\rho(x)$ is the topological charge density, $\rho(x)=\frac{1}{32 \pi^{2}} \varepsilon_{\mu \nu \lambda \sigma} \operatorname{tr}\left[F_{\mu \nu}(x) F_{\lambda \sigma}(x)\right]$. Using the chiral perturbation theory (ChPT), Leutwyler and Smilga [1] obtained the following relation at the tree level,

$$
\chi_{t}= \begin{cases}\Sigma\left(m_{u}^{-1}+m_{d}^{-1}\right)^{-1}, & N_{f}=2 \\ \Sigma\left(m_{u}^{-1}+m_{d}^{-1}+m_{s}^{-1}\right)^{-1}, & N_{f}=3\end{cases}
$$

where $m_{u}, m_{d}$, and $m_{s}$ are the quark masses, and $\Sigma$ is the chiral condensate. This implies that in the chiral limit $\left(m_{u} \rightarrow 0\right)$, the topological susceptibility is suppressed due to internal quark loops. Most importantly, (1.2) provides a viable way to extract $\Sigma$ from $\chi_{t}$ in the chiral limit. Recently, the topological susceptibility has been derived to the one-loop order in ChPT for an arbitrary number of flavors $[2,3]$. It would be interesting to see whether the $\chi_{t}$ measured in lattice QCD with exact chiral symmetry would agree with ChPT to the one-loop order. This will be addressed in our forthcoming paper. Besides $\chi_{t}$, it is also interesting to determine the second normalized cumulant,

$$
c_{4}=-\frac{1}{\Omega}\left[\left\langle Q_{t}^{4}\right\rangle-3\left\langle Q_{t}^{2}\right\rangle^{2}\right]
$$

which is related to the leading anomalous contribution to the $\eta^{\prime}-\eta^{\prime}$ scattering amplitude in QCD, as well as the dependence of the vacuum energy on the vacuum angle $\theta$. Recently $c_{4}$ has been derived in ChPT (at the tree-level) for an arbitrary number of flavors [2, 3],

$$
c_{4}= \begin{cases}-\Sigma\left(m_{u}^{-3}+m_{d}^{-3}\right)\left(m_{u}^{-1}+m_{d}^{-1}\right)^{-4}, & N_{f}=2, \\ -\Sigma\left(m_{u}^{-3}+m_{d}^{-3}+m_{s}^{-3}\right)\left(m_{u}^{-1}+m_{d}^{-1}+m_{s}^{-1}\right)^{-4}, & N_{f}=3 .\end{cases}
$$

In this work, we investigate to what extent $\chi_{t}$ and $c_{4}$ in lattice QCD with exact chiral symmetry would agree with the ChPT at the tree level, Eqs. (1.2) and (1.4), and determine $\Sigma$ from our data of $\chi_{t}$. In principle, one can also extract $\Sigma$ from $c_{4}$, however, this would require much higher statistics than that of $\chi_{t}$.

From Eqs. (1.1) and (1.3), $\chi_{t}$ and $c_{4}$ can be determined by counting the number of gauge configurations in each topological sector. Obviously, if one fixes the topology at the trivial sector with $Q_{t}=0$, then $\chi_{t}=c_{4}=0$. However, even for a topologically-trivial gauge configuration, it may possess non-trivial topological excitations in sub-volumes. Thus, one can still measure $\chi_{t}$ and $c_{4}$ using the correlation of the topological charges of sub-volumes. In general, for any topological sector with $Q_{t}$, using saddle point expansion on the QCD partition function in a finite volume, it can be shown that [4]

$$
\lim _{|x| \rightarrow \infty}\langle\rho(x) \rho(0)\rangle=\frac{1}{\Omega}\left(\frac{Q_{t}^{2}}{\Omega}-\chi_{t}-\frac{c_{4}}{2 \chi_{t} \Omega}\right)+\mathscr{O}\left(\Omega^{-3}\right) .
$$


However, it is difficult to extract $\rho(x)$ and $Q_{t}$ unambiguously from the gauge link variables, due to their rather strong fluctuations.

To circumvent this difficulty, one may consider the Atiyah-Singer index theorem [5]

$$
Q_{t}=n_{+}-n_{-}=\operatorname{index}(\mathscr{D})
$$

where $n_{ \pm}$is the number of zero modes of the massless Dirac operator $\mathscr{D} \equiv \gamma_{\mu}\left(\partial_{\mu}+i g A_{\mu}\right)$ with \pm chirality. Since $\mathscr{D}$ is anti-Hermitian and chirally symmetric, its nonzero eigenmodes must come in complex conjugate pairs with zero chirality. Thus one can obtain the identity

$$
n_{+}-n_{-}=\int d^{4} x m \operatorname{tr}\left[\gamma_{5}(\mathscr{D}+m)^{-1}(x, x)\right]
$$

by spectral decomposition. In view of (1.6) and (1.7), one can regard $m \operatorname{tr}\left[\gamma_{5}(\mathscr{D}+m)^{-1}(x, x)\right]$ as topological charge density, to replace $\rho(x)$ in the measurement of $\chi_{t}$.

For lattice QCD, it is well-known that the overlap Dirac operator [6, 7] in a topologically non-trivial gauge background possesses exact zero modes (with definite chirality) satisfying the Atiyah-Singer index theorem. Thus the topological charge density can be defined as

$$
\rho_{m}(x)=m \operatorname{tr}\left[\gamma_{5}\left(D_{c}+m\right)_{x, x}^{-1}\right],
$$

where $\left(D_{c}+m\right)^{-1}$ is the valence quark propagator with quark mass $m$. Here $\rho_{m}(x)$ is justified to be topological charge density, since it can be shown that $\sum_{x} \rho_{m}(x)=n_{+}-n_{-}$, which is similar to its counterpart in continuum, (1.7). However, on a finite lattice, it is contaminated by $m_{\pi}, m_{\eta^{\prime}}$ and any states which can couple to $\left\langle\rho_{m}(x) \rho_{m}(0)\right\rangle$. An alternative is to consider the correlator of the flavor-singlet pseudoscalar meson $\eta^{\prime}[4]$

$$
\lim _{\left|x_{1}-x_{2}\right| \gg 1} m_{q}^{2}\left\langle\eta^{\prime}\left(x_{1}\right) \eta^{\prime}\left(x_{2}\right)\right\rangle_{Q}=-\frac{\chi_{t}}{\Omega}\left(1-\frac{Q^{2}}{\chi_{t} \Omega}+\frac{c_{4}}{2 \chi_{t}^{2} \Omega}\right)+\mathscr{O}\left(e^{-m_{\eta^{\prime}}\left|x_{1}-x_{2}\right|}\right)+\mathscr{O}\left(\Omega^{-3}\right)
$$

which is equal to the disconnected part $\left\langle\rho_{m}\left(x_{1}\right) \rho_{m}\left(x_{2}\right)\right\rangle_{Q}$ at large separation, but it tends to the asymptotic value faster than the latter since it only couples to the states containing $\eta^{\prime}$. Then the time-correlation function of $\eta^{\prime}$ is fitted to $A+B\left(e^{-M t}+e^{-M(T-t)}\right)$ to obtain the constant $A=$ $\frac{1}{m_{q}^{2}} \frac{1}{T}\left(\frac{Q_{t}^{2}}{\Omega}-\chi_{t}-\frac{c_{4}}{2 \chi_{t} \Omega}\right)$, and from which to extract $\chi_{t}$ provided that $\left|c_{4}\right| \ll 2 \chi_{t}^{2} \Omega$. This was how we determined the topological susceptibility in two-flavor lattice QCD with fixed topology [8,9].

However, it was unclear to what extent the assumption $\left|c_{4}\right| \ll 2 \chi_{t}^{2} \Omega$ was satisfied. To eliminate this constraint, we compute the 4-point correlator of $\eta^{\prime}$, as well as the 2-point correlator. Theoretically, in a fixed topology, the former behaves as [4]

$$
\lim _{\left|x_{i}-x_{j}\right| \gg 1} m_{q}^{4}\left\langle\eta^{\prime}\left(x_{1}\right) \cdots \eta^{\prime}\left(x_{4}\right)\right\rangle_{Q}=\frac{3 \chi_{t}^{2}}{\Omega^{2}}\left(1-\frac{Q^{2}}{\chi_{t} \Omega}+\frac{c_{4}}{\chi_{t}^{2} \Omega}\right)^{2}+\mathscr{O}\left(e^{-m_{\eta^{\prime}}\left|x_{i}-x_{j}\right|}\right)+\mathscr{O}\left(\Omega^{-4}\right) .
$$

From (1.9) and (1.10), one can solve for $\chi_{t}$ and $c_{4}$ (or equivalently, the parameter $y$ )

$$
\begin{aligned}
\chi_{t} & =\frac{Q^{2}}{\Omega}+\Omega\left(2 k_{2}-\sqrt{k_{4} / 3}\right), \\
y & \equiv \frac{c_{4}}{2 \chi_{t}^{2} \Omega}=-\frac{\left(\sqrt{k_{4} / 3}-k_{2}\right)}{\sqrt{k_{4} / 3}-2 k_{2}}\left(1-\frac{Q^{2}}{\chi_{t} \Omega}\right),
\end{aligned}
$$


where $-k_{2}$ and $k_{4}$ are the asymptotic values of 2-point and 4-point correlators at large separation. It is interesting to note that if one neglects the $y$ term in (1.9) and (1.10), they reduce to

$$
\chi_{t} \simeq \frac{Q^{2}}{\Omega}+\Omega k_{2}, \quad \chi_{t} \simeq \frac{Q^{2}}{\Omega}+\Omega \sqrt{k_{4} / 3},
$$

which provide two independent estimates of $\chi_{t}$. In other words, if $|y| \ll 1$, then (1.11) and (1.13) all give compatible results for $\chi_{t}$.

At this point, we note that (1.9) and (1.10) are only applicable for all flavors having the same mass $m_{q}$. To remedy this problem, we introduce the "topological" $\eta^{\prime}$ interpolating operator

$$
\eta_{T}^{\prime}(x)=\frac{1}{N_{f}} \sum_{f=1}^{N_{f}} m_{f} \overline{\mathrm{q}}^{f}(x) \gamma_{5} \mathrm{q}^{f}(x),
$$

and transcribe (1.9) and (1.10) to

$$
\begin{aligned}
\lim _{\left|x_{1}-x_{2}\right| \gg 1}\left\langle\eta_{T}^{\prime}\left(x_{1}\right) \eta_{T}^{\prime}\left(x_{2}\right)\right\rangle_{Q} & =-\frac{\chi_{t}}{\Omega}\left(1-\frac{Q^{2}}{\chi_{t} \Omega}+\frac{c_{4}}{2 \chi_{t}^{2} \Omega}\right)+\cdots \\
\lim _{\left|x_{i}-x_{j}\right| \gg 1}\left\langle\eta_{T}^{\prime}\left(x_{1}\right) \eta_{T}^{\prime}\left(x_{2}\right) \eta_{T}^{\prime}\left(x_{3}\right) \eta_{T}^{\prime}\left(x_{4}\right)\right\rangle_{Q} & =\frac{3 \chi_{t}^{2}}{\Omega^{2}}\left(1-\frac{Q^{2}}{\chi_{t} \Omega}+\frac{c_{4}}{\chi_{t}^{2} \Omega}\right)^{2}+\cdots
\end{aligned}
$$

The time-correlation functions of 2-point and 4-point correlators of $\eta_{T}^{\prime}$ can be measured as

$$
\begin{aligned}
C_{\eta_{T}^{\prime}}(t) & =\frac{1}{L^{3} T} \sum_{u=1}^{T} \sum_{\vec{x}_{i}}\left\langle\eta_{T}^{\prime}\left(\vec{x}_{2}, u+t\right) \eta_{T}^{\prime}\left(\vec{x}_{1}, u\right)\right\rangle \\
C_{4 \eta_{T}^{\prime}}(t) & =\frac{1}{L^{3} T} \sum_{u=1}^{T} \sum_{\vec{x}_{i}}\left\langle\eta_{T}^{\prime}\left(\vec{x}_{4}, u+3 t\right) \eta_{T}^{\prime}\left(\vec{x}_{3}, u+2 t\right) \eta_{T}^{\prime}\left(\vec{x}_{2}, u+t\right) \eta_{T}^{\prime}\left(\vec{x}_{1}, u\right)\right\rangle .
\end{aligned}
$$

At large $t$, their asymptotic values are $-k_{2}$ and $k_{4}$, which are inserted into (1.11) and (1.12) to obtain $\chi_{t}$ and $c_{4}$.

\section{Lattice Setup}

Our simulations are caried out on the lattices $16^{3} \times 32\left(N_{f}=2\right)$ and $16^{3} \times 48\left(N_{f}=2+1\right)$, at a lattice spacing $\sim 0.11 \mathrm{fm}[10,11]$. For the gluon part, we use the Iwasaki action at $\beta=$ 2.30, together with unphysical Wilson fermions and associated twisted-mass ghosts [12]. The unphysical degrees of freedom generate a factor $\operatorname{det}\left[H_{w}^{2}\left(-m_{0}\right) /\left(H_{w}^{2}\left(-m_{0}\right)+\mu^{2}\right)\right]$ in the partition function (with $m_{0}=1.6$ and $\mu=0.2$ ) that suppresses the near-zero eigenvalue of $H_{w}\left(-m_{0}\right)$ and thus makes the numerical operation with the overlap operator substantially faster. Furthermore, since the exact zero eigenvalue is forbidden, the global topological change is preserved during the molecular dynamics evolution of the gauge field.

We choose the sea quark masses $m_{u}$ in the range $m_{s} / 6-m_{s}$. For $N_{f}=2$, we take six sea quark mass $m_{u(d)}$ values: $0.015,0.025,0.035,0.050,0.070$, and 0.100 , while for $N_{f}=2+1$ with $m_{s}=0.080 / 0.100$, we take five sea quark mass $m_{u(d)}$ values: $0.015,0.025,0.035,0.050$, and $0.080 / 0.100$. After discarding 500 trajectories for thermalization, we accumulate $10000\left(N_{f}=\right.$ 




(a)

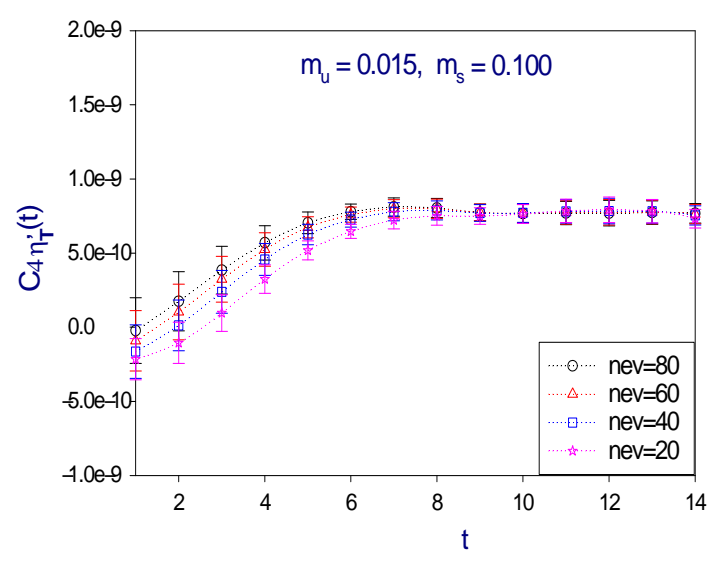

(b)

Figure 1: Low-mode saturation of (a) the 2-point function $C_{\eta_{T}^{\prime}}(t)$ (b) the 4-point function $C_{4 \eta_{T}^{\prime}}(t)$

2) $/ 2500\left(N_{f}=2+1\right)$ trajectories in total for each sea quark mass. In the calculation of $\chi_{t}$, we take one configuration every $20\left(N_{f}=2\right) / 5\left(N_{f}=2+1\right)$ trajectories, thus we have 500 configurations for each $m_{q}$. For each configuration, $50\left(N_{f}=2\right) / 80\left(N_{f}=2+1\right)$ pairs of lowest-lying eigenmodes of the overlap-Dirac operator $D(0)$ are calculated using the implicitly restarted Lanczos algorithm and stored for the later use. In these calculations, the better accuracy of $O\left(10^{-12}\right)$ is enforced for the sign function by increasing the number of poles in the rational approximation.

\section{Results}

In practice, we use 50/80 pairs of low-lying eigenmodes of the overlap operator to evaluate the 2-point and 4-point time-correlation functions of $\eta_{T}^{\prime}$. Thus it is crucial to check whether these low-lying eigenmodes suffice to saturate $C_{\eta_{T}^{\prime}}(t)$ and $C_{4 \eta_{T}^{\prime}}(t)$ respectively. In Fig. 1, we plot $C_{\eta_{T}^{\prime}}(t)$ and $C_{4 \eta_{T}^{\prime}}(t)$ for $m_{u} a=0.015$ and $m_{s} a=0.100$, versus the number of eigenmodes (nev) 20, 40, 60, and 80 respectively. Obviously, both $C_{\eta_{T}^{\prime}}(t)$ and $C_{4 \eta_{T}^{\prime}}(t)$ are well saturated with 80 eigenmodes for the time range $15 \leq t \leq 24$ and $9 \leq t \leq 14$ respectively. The low-mode saturation also holds for all sea quark masses, for $N_{f}=2$ and $N_{f}=2+1$.

In Figs. 2-4, we plot the values of $a^{4} \chi_{t}$ and $a^{4} c_{4}$ versus the sea quark mass $m_{q} a$, together with the values of $\chi_{t}$ obtained from the 2-point and 4-point functions (1.13) respectively. Evidently, the values of $\chi_{t}$ from (1.11) and (1.13) are in good agreement with one another.

For the smallest four quark masses, $0.015,0.025,0.035$, and 0.050 , the data points of $a^{4} \chi_{t}$ are well fitted by the Leutwyler-Smilga formula (1.2). Our results of $a^{3} \Sigma$ are:

$$
a^{3} \Sigma= \begin{cases}0.00299(9), & N_{f}=2 \\ 0.00227(8), & N_{f}=2+1\left(m_{s}=0.080\right) \\ 0.00222(10), & N_{f}=2+1\left(m_{s}=0.100\right)\end{cases}
$$

In order to convert $\Sigma$ to that in the $\overline{\mathrm{MS}}$ scheme, we calculate the renormalization factor $Z_{m}^{\overline{\mathrm{MS}}}(2 \mathrm{GeV})$ using the non-perturbative renormalization technique through the RI/MOM scheme, 


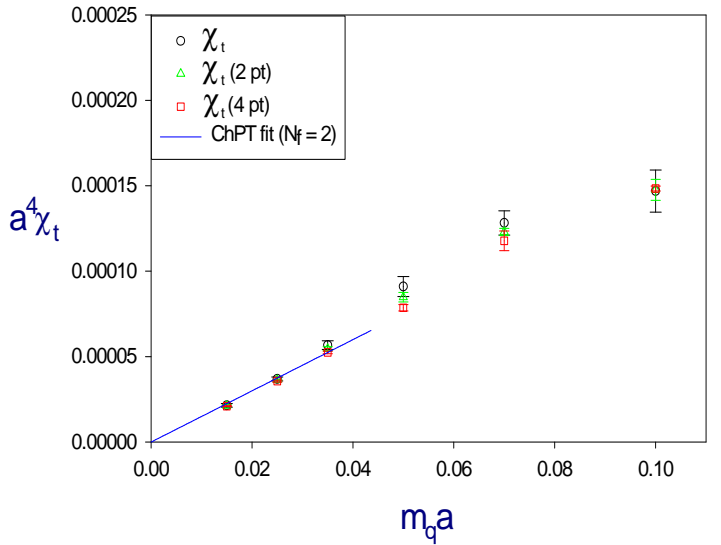

(a)

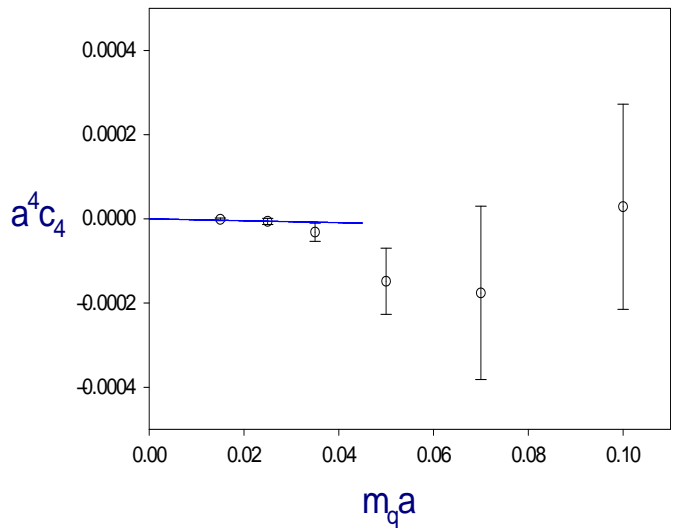

(b)

Figure 2: Topological susceptibility $a^{4} \chi_{t}$ and $a^{4} c_{4}$ versus sea quark mass $m_{q} a$ for 2-flavor lattice QCD with fixed topological charge $Q_{t}=0$.

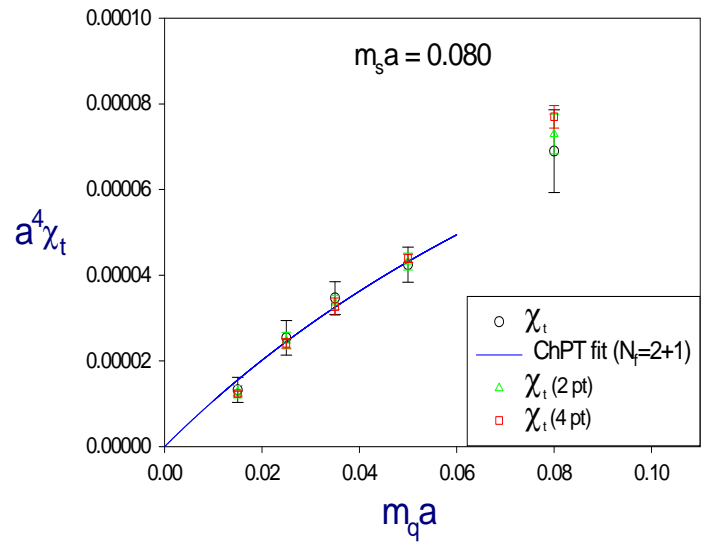

(a)

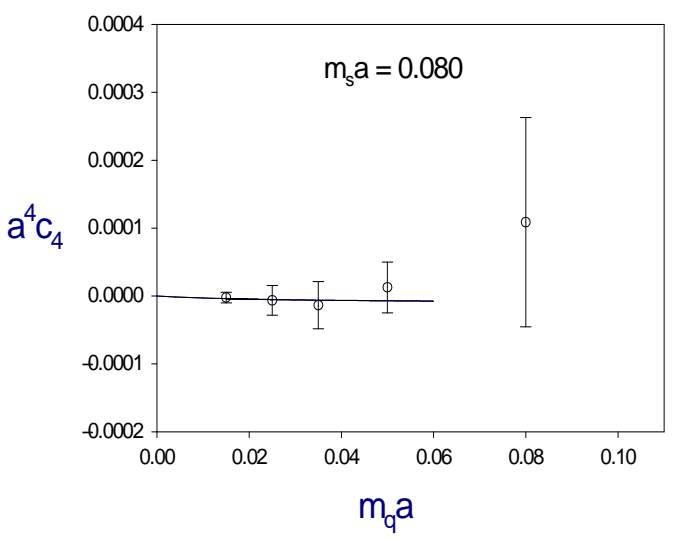

(b)

Figure 3: Topological susceptibility $a^{4} \chi_{t}$ and $a^{4} c_{4}$ versus sea quark mass $m_{q} a$ for (2+1)-flavor lattice QCD with fixed topological charge $Q_{t}=0$, for $m_{s}=0.080$.

and our results are [13]

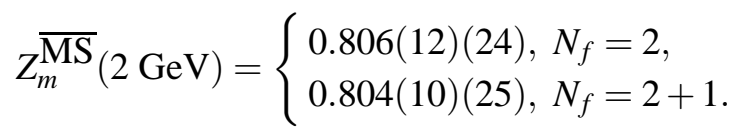

With $a^{-1}=1670(40) \mathrm{MeV}\left(N_{f}=2\right)$ and $a^{-1}=1833(12) \mathrm{MeV}\left(N_{f}=2+1\right)$ determined with $r_{0}=0.49 \mathrm{fm}[11]$, the values of $\Sigma$ are transcribed to

$$
\left[{ }_{\Sigma} \overline{\mathrm{MS}}_{(2 \mathrm{GeV})}\right]^{1 / 3}=\left\{\begin{array}{l}
259(7)(8) \mathrm{MeV}, N_{f}=2, \\
259(7)(6) \mathrm{MeV}, N_{f}=2+1\left(m_{s}=0.080\right), \\
258(8)(7) \mathrm{MeV}, N_{f}=2+1\left(m_{s}=0.100\right),
\end{array}\right.
$$




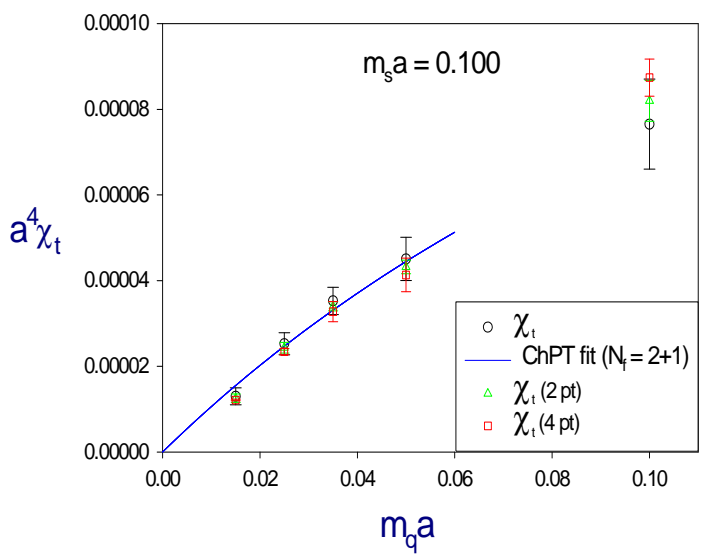

(a)



(b)

Figure 4: Topological susceptibility $a^{4} \chi_{t}$ and $a^{4} c_{4}$ versus sea quark mass $m_{q} a$ for (2+1)-flavor lattice QCD with fixed topological charge $Q_{t}=0$, for $m_{s}=0.100$.

which are in good agreement with that extracted from $\chi_{t}=\left\langle Q_{t}^{2}\right\rangle / \Omega$ with $Q_{t}$ determined by the spectral flow method for the $2+1$ flavors QCD configurations generated by the RBC and UKQCD Collaborations with domain-wall fermion [14]. Also, they are in good agreement with our previous results extracted from $\chi_{t}$ in 2-flavor QCD $[8,9]$, and from the low-lying eigenvalues in the $\varepsilon$-regime $[16,15]$. The errors represent a combined statistical error $\left(a^{-1}\right.$ and $\left.Z_{m}^{\overline{\mathrm{MS}}}\right)$ and the systematic error respectively. Since the calculation is done at a single lattice spacing, the discretization error cannot be quantified reliably, but we do not expect much larger error because our lattice action is free from $O(a)$ discretization effects.

Next we turn to the second normalized cumulant $\left(c_{4}\right)$. For the smallest four (three for the case $N_{f}=2$ ) quark masses, $0.015,0.025,0.035$, and 0.050 , the data points of $a^{4} c_{4}$ can be fitted by the ChPT formula (1.4) at the tree level. Our results of $a^{3} \Sigma$ are:

$$
a^{3} \Sigma=\left\{\begin{array}{l}
0.0018(16), \quad N_{f}=2, \\
0.0030(9), \quad N_{f}=2+1\left(m_{s}=0.080\right), \\
0.0025(12), \quad N_{f}=2+1 \quad\left(m_{s}=0.100\right),
\end{array}\right.
$$

which are consistent with those (3.1) extracted from $\chi_{t}$, in spite of very large error bars.

\section{Concluding remark}

In this work, we obtain the topological susceptibility $\chi_{t}$ and the second normalized moment $c_{4}$, in 2-flavor and (2+1)-flavor QCD, from a lattice calculation of 2-point and 4-point correlators at a fixed global topological charge $Q_{t}=0$. In the small $m_{q}$ regime, our results of $\chi_{t}$ and $c_{4}$ both agree with ChPT (at the tree level). This asserts that the topologically non-trivial excitations are in fact locally active in the QCD vacuum, even when the global topological charge is zero. We will use the values of $\chi_{t}$ we have determined to remove the artifacts in any physical observables due to the fixed topology in a finite volume $[17,4]$. Our next task is to investigate to what extent our results of $\chi_{t}$ would agree with the ChPT to the one-loop order $[2,3]$. 


\section{Acknowledgments}

Numerical simulations are performed on Hitachi SR11000 and IBM System Blue Gene Solution at High Energy Accelerator Research Organization (KEK) under a support of its Large Scale Simulation Program (No. 09-05), and also on IBM and HP clusters at NCHC and NTU-CC in Taiwan. This work is supported in part by the Grant-in-Aid of the Japanese Ministry of Education (Nos. 21674002, 18740167, 20025010, 19540286, 19740160, 20105005, 19540286, 20105002, 21684013, 20340047, 20105001, 20105003), GCOE of Nagoya Univ, QFPU of JSPS, the National Science Council of Taiwan (Nos. NSC96-2112-M-002-020-MY3, NSC96-2112-M-001-017-MY3, NSC98-2119-M-002-001), and NTU-CQSE (Nos. 98R0066-65, 98R0066-69).

\section{References}

[1] H. Leutwyler and A. Smilga, Phys. Rev. D 46, 5607 (1992).

[2] Y. Y. Mao and T. W. Chiu [TWQCD Collaboration], Phys. Rev. D 80, 034502 (2009) [arXiv:0903.2146 [hep-lat]].

[3] S. Aoki and H. Fukaya, arXiv:0906.4852 [hep-lat].

[4] S. Aoki, H. Fukaya, S. Hashimoto and T. Onogi, Phys. Rev. D 76, 054508 (2007)

[5] M. F. Atiyah and I. M. Singer, Annals Math. 87, 484 (1968).

[6] H. Neuberger, Phys. Lett. B 417, 141 (1998); Phys. Lett. B 427, 353 (1998)

[7] R. Narayanan and H. Neuberger, Nucl. Phys. B 443, 305 (1995)

[8] T. W. Chiu et al. [JLQCD and TWQCD Collaborations], PoS LAT2007, 068 (2007)

[9] S. Aoki et al. [JLQCD and TWQCD Collaborations], Phys. Lett. B 665, 294 (2008)

[10] S. Hashimoto et al. [JLQCD collaboration], PoS LAT2007, 101 (2007)

[11] H. Matsufuru et al. [JLQCD and TWQCD Collaborations], PoS LAT2008, 077 (2008)

[12] H. Fukaya, S. Hashimoto, K. I. Ishikawa, T. Kaneko, H. Matsufuru, T. Onogi and N. Yamada [JLQCD Collaboration], Phys. Rev. D 74, 094505 (2006)

[13] J. Noaki et al., arXiv:0907.2751 [hep-lat].

[14] T. W. Chiu, T. H. Hsieh and P. K. Tseng [TWQCD Collaboration], Phys. Lett. B 671, 135 (2009)

[15] H. Fukaya et al. [JLQCD Collaborations], Phys. Rev. Lett. 98, 172001 (2007)

[16] H. Fukaya et al. [JLQCD and TWQCD Collaborations], Phys. Rev. D 76, 054503 (2007)

[17] R. Brower, S. Chandrasekharan, J. W. Negele and U. J. Wiese, Phys. Lett. B 560, 64 (2003) 